\title{
Hybrid Computational Techniques for Electromagnetic Propagation Analysis in Complex Indoor Environments
}

\begin{tabular}{|r|l|}
\hline Journal: & IEEE Antennas and Propagation Magazine \\
\hline Manuscript ID & APM-Jun-2018-0106 \\
\hline Manuscript Type: & Original Article \\
\hline Date Submitted by the Author: & 21 -Jun-2018 \\
\hline Complete List of Authors: & $\begin{array}{l}\text { Azpilicueta, Leyre; Instituto Tecnologico y de Estudios Superiores de } \\
\text { Monterrey, } \\
\text { Falcone, Francisco } \\
\text { Janaswamy, Ramakrishna; University of Massachusetts }\end{array}$ \\
\hline Keywords: & $\begin{array}{l}\text { 3D-Ray Launching, Diffusion, Scattering, RF environment modeling, radio } \\
\text { channel simulation }\end{array}$ \\
\hline
\end{tabular}




\title{
Hybrid Computational Techniques for Electromagnetic Propagation Analysis in Complex Indoor Environments
}

\author{
Leyre Azpilicueta, Member, IEEE, Francisco Falcone, Senior Member, IEEE, \\ and Ramakrishna Janaswamy, Fellow, IEE
}

\begin{abstract}
In this work, the comparison of different deterministic methodologies to characterize channel behavior in heterogeneous and composite scenarios is presented. These techniques combine a 3-D Ray Launching approach based on Geometrical Optics, with other approaches based on Geometrical Optics and Uniform Theory of Diffraction, and a full technique, which also includes a Diffusion Equation method based on the equation of transfer. A new methodology based on Geometrical Optics and Diffusion Equation is presented and validated when compared with real measurements, achieving accurate results. The proposed technique provides a computational time reduction of up to $90 \%$ with respect to the conventional Geometrical Optics with Uniform Theory of Diffraction and Diffusion Equation approach.
\end{abstract}

Index Terms - 3D-Ray Launching, Diffusion, scattering, RF environment modeling, radio channel simulation.

\section{INTRODUCTION}

$\mathrm{W}$ ith the growing demand of wireless communications systems over the last years, the need of having efficient tools to characterize electromagnetic propagation in different complex environments has increased significantly. The capacity of defining the best choices for the locations of the transceivers in a wireless communication system, estimating their coverage/capacity relations not having to carry out campaigns of real measurements, which are usually quite expensive and time absorbing, has been a requirement in the past years. Because of that, it has become necessary to develop efficient and accurate new prediction propagation approaches which help with the design of wireless communication systems and networks. In this way, radio planning as well as system-level design and optimization tasks can be performed, such as coverage/capacity analysis in HetNet operation, or the energy efficiency analysis in wireless sensor networks [1].

Empirical methods were the first methods used for initial coverage prediction [2-3], i.e. COST-231, Walfish-Bertoni or

Leyre Azpilicueta is with the School of Engineering and Sciences of Tecnologico de Monterrey, Mexico (e-mail: leyre.azpilicueta@itesm.mx).

Francisco Falcone is with the Electrical and Electronic Engineering Dept, Universidad Pública de Navarra, Pamplona, Navarra, Spain (phone: +34-948169741; fax: +34-948-160720; e-mail: francisco.falcone@unavarra.es).

Ramakrishna Janaswamy is with the Department of Electrical and Computer Engineering, University of Massachusetts at Amherst, Amherst, MA 01003 USA (e-mail: janaswamy@ecs.umass.edu).
Okumura Hata. Their benefit is that they are fast, in terms of computational time needed for simulation, but the disadvantage is that results need to be calibrated based on real measurements with linear regression methods.

On the other hand, deterministic methods [4-5] can be divided in two groups, being the first one those methods based on full-wave simulation approaches, i.e. method of moment (MoM) or finite difference time domain (FDTD) [6]. The second group are those methods based on geometrical approximations, such as ray launching (RL) and ray tracing (RT) [7]. The advantage of these methods is the precision of the results, but the drawback is that the simulation computational time can be unaffordable if the analyzed scenario is complex and with large dimensions. They usually are used combined with the uniform theory of diffraction (UTD) to predict radio coverage [8-9]. It has been shown in the literature that RT and RL approaches achieve a trade-off between accuracy and computational time, and because of that, they are the most used for multipath propagation prediction in urban and complex indoor environments.

It has been shown in the literature the dispersive behavior of electromagnetic waves in a discrete random medium with sufficient multiple scattering [10-11]. Taking this into account, it is highly advantageous to consider the diffuse scattering when assessing wireless electromagnetic channel performance.

There are several works which have implemented this phenomenon in deterministic approaches. In [12], a diffuse scattering implementation in a three-dimensional (3D) urban propagation environment has been described. The influence of diffuse scattering when analyzing narrowband and wideband characteristics is presented in [13-14], which present a field prediction technique which takes into account reflection, diffraction and diffuse scattering, showing that diffuse scattering plays an important role in electromagnetic propagation. In [15], a novel and efficient hybrid model combining a two-dimensional (2D) site-specific model and a statistical model is presented, modeling the presented mean addition of diffused scattering.

Nevertheless, these mentioned approaches could have an unaffordable computational complexity which is determined by the results exactness. Nowadays, reducing simulation computational time has become a challenge and, in order to overwhelmed this disadvantage, different speed-up approaches 
have been proposed in the literature. Reference [16] presents a novel deterministic approach to model radio wave propagation channel, which permit the utilization of less number of transmitted rays in the simulation scenario, whereas intermediate points can be predicted using neural network. In [17] several acceleration techniques to improve the storage of data and processing are shown. Some authors have worked on acceleration techniques by decomposing the $3 \mathrm{D}$ problem into two 2D sub-problems [18-19], whereas in [20] the methodology of splitting the 3D wave propagation into $2 \mathrm{D}$ planes is shown. In [21-22], the medium is tessellated using rectangular and triangular meshes, respectively. In [23], a database preprocessing and discretization of the environment is proposed. Finally, with the aim of cutting down the high quantity of rays and the huge input database handled by the algorithm, two methods are presented in [24].

In the light of the above analysis of diverse propagation approaches, it is extremely important to consider deterministic models which convey with precise results with an accessible simulation computational time. In this sense, an analysis of different deterministic approaches has been done in this work, leading to the conclusion that a novel and efficient hybrid Geometrical Optics-Diffusion Equation (GO/DE) methodology to assess electromagnetic propagation in heterogeneous and composite indoor scenarios achieves the best results in terms of accuracy and simulation computational time. The proposed approach has been presented in the letter [25]. It is an acceleration technique combining the full GO with the Uniform Theory of Diffraction and DE (GO/UTD/DE). In this work, different realistic scenarios in which the different approaches are applied are presented. The novel technique is validated showing accurate results and it is computationally more efficient when compared to the fully $\mathrm{GO} / \mathrm{UTD} / \mathrm{DE}$ technique, with a rise in a mean error of $0.27 \mathrm{~dB}$. Fig. 1 describes the presented work, showing the advantages and disadvantages of each one of the cases which have been analyzed.

The paper is organized as follows. In Section II, the RL method is analytically described with the modeling of edge contributions. Section III presents the DE approach and its implementation into the RL method. Simulation results are discussed in Section IV and, in Section V, measurement results and processing gain in terms of computational time are reported. Finally, conclusions are given in Section VI.

\section{RAY LAUNCHING APPROACH}

In order to perform wireless channel analysis, a 3-D RL algorithm has been developed in-house on Geometrical Optics (GO) and its extension the Geometrical Theory of Diffraction (GTD). The main principle of RL techniques is that, based on an addition of optic and electromagnetic theories, the radiated wave is approximated as a set of rays which propagate along the space.

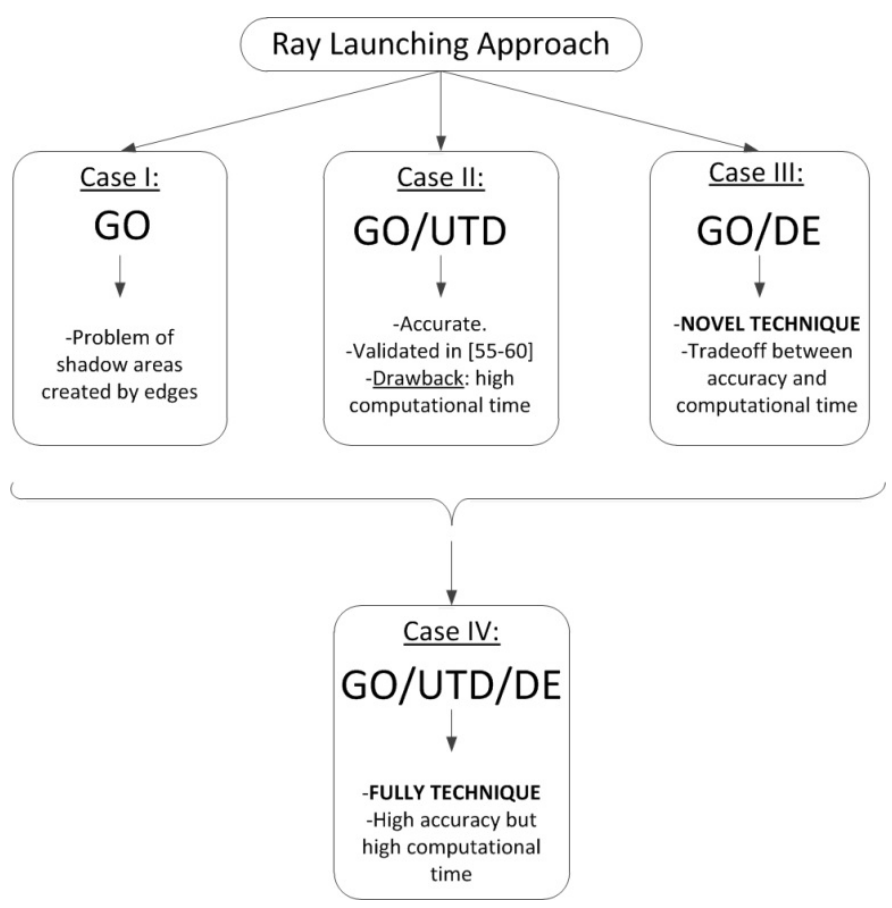

Fig. 1. Scheme with the different deterministic approaches considered with the benefits and drawbacks of each one.

Rays are launched as defined in the spherical coordinate system at an elevation angle $\theta$ and an azimuth angle $\phi$. The parameters of the antenna, as well as the radiation pattern are taken into account. Parameters such as frequency of operation, number of multipath reflections, angular resolution, and cuboids dimension are introduced.

Each ray propagates in the space as a single optical wavefront. The electric field $E$ created by an antenna with a radiated power $P_{\text {rad }}$ with a directivity $D_{t}\left(\theta_{t}, \phi_{t}\right)$ and polarization ratio $\left(X^{\perp}, X^{\|}\right)$at a distance $r$ in the free space is calculated by [26]

$$
\begin{gathered}
E_{i}^{\perp}=\sqrt{\frac{P_{r a d} D_{t}\left(\theta_{t}, \phi_{t}\right) \eta_{0}}{2 \Pi}} \frac{e^{-j \beta_{0} r}}{r} X^{\perp} L^{\perp} \\
E_{i}^{\|}=\sqrt{\frac{P_{r a d} D_{t}\left(\theta_{t}, \phi_{t}\right) \eta_{0}}{2 \Pi}} \frac{e^{-j \beta_{0} r}}{r} X^{\|} L^{\|}
\end{gathered}
$$

where $\quad \beta_{0}=2 \pi f_{c} \sqrt{\varepsilon_{0} \mu_{0}}, \quad \varepsilon_{0}=8.854 * 10^{-12} \mathrm{~F} / \mathrm{m}, \quad \mu_{0}=4 \pi * 10^{-7}$ $\mathrm{H} / \mathrm{m}$ and $\eta_{0}=120 \pi$ ohms. $f_{c}$ is the transmission frequency and $L^{\perp \|}$ are the path loss coefficients for each polarization.

When this ray finds an object in its path, two new rays are created: a reflected ray and a transmitted ray. These rays have new angles provided by Snell's law [27].

It is important to emphasize that a full $3 \mathrm{D}$ scenario is created before simulation in which objects, walls, transmitters, receivers and the complete set of elements within the simulation environment are considered. It must be pointed out a volumetric grid is implemented storing the different parameters and characteristics of each ray propagating in the space. In consequence, the whole area is separated into several 
cuboids of fixed dimensions. When a ray enters a specific cuboid, its parameters are saved in a matrix. Dispersive material properties within the considered frequency range for all the elements within the scenario are also taken into account.

\section{A. Ray Launching Modeling with Edge Contributions}

Nevertheless, one of the main problems that presents the GO only approach is that it cannot estimate correctly the received field in shadow areas originated by edges or discontinuities of the obstacles in the scenario, as it is represented in Fig. 2. The reason is that only the direct, reflected and refracted rays are taking into account in the GO only approach, heading to the presence of unexpected areas, which correspond to the boundaries where these rays exist. Because of that, diffraction phenomenon was introduced first by Keller [28]. He stated that when GO rays leave the edge of the obstacles, they were diffracted rays, and they follow the rules of the generalized Fermat's principle [29]. However, where the GO rays present discontinuities, this formulation had inaccuracies in shadow and reflection regions. To solve these problems, uniform solutions to the asymptotic expressions were used. The first one is the uniform theory of diffraction (UTD) developed by Kouyoumjian and Pathak [30] from the asymptotic expansion by Pauli Clemmow [31], whereas the second one is the uniform asymptotic solution (UAT) obtained by Lee and Deschamps [32]. The UAT obtains the fields by removing two terms with infinite values at the shadow borders, while the UTD is numerically easier to be implemented.

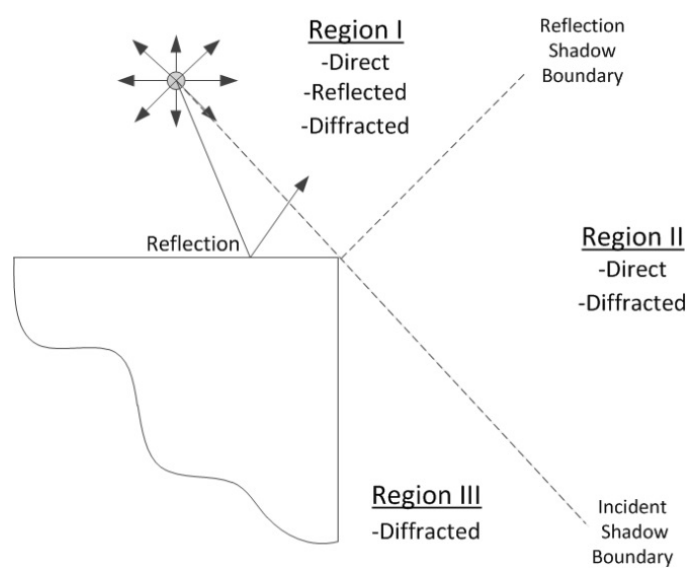

Fig. 2. Illustration of the regions described by GO rays.

GO/UTD approach has been widely used to predict mobile communication propagation for both urban [33-34] and indoor [35-36] environments. In the in-house developed software, the UTD has been implemented by computing the diffraction coefficients on the edges of the diffractive elements with the finite conductivity two-dimensional diffraction coefficients given by [37] as

$$
D^{\| \perp}=\frac{-e^{(-j \pi / 4)}}{2 n \sqrt{2 \pi k}}\left\{\begin{array}{c}
\cot \left(\frac{\pi+\left(\Phi_{2}-\Phi_{1}\right)}{2 n}\right) F\left(k L a^{+}\left(\Phi_{2}-\Phi_{1}\right)\right) \\
+\cot \left(\frac{\pi-\left(\Phi_{2}-\Phi_{1}\right)}{2 n}\right) F\left(k L a^{-}\left(\Phi_{2}-\Phi_{1}\right)\right) \\
+R_{0}^{\| \perp} \cot \left(\frac{\pi-\left(\Phi_{2}+\Phi_{1}\right)}{2 n}\right) F\left(k L a^{-}\left(\Phi_{2}+\Phi_{1}\right)\right) \\
+R_{n}^{\| \perp} \cot \left(\frac{\pi+\left(\Phi_{2}+\Phi_{1}\right)}{2 n}\right) F\left(k L a^{+}\left(\Phi_{2}+\Phi_{1}\right)\right)
\end{array}\right\}
$$

where $\mathrm{n} \pi$ is the wedge angle, $\mathrm{F}, \mathrm{L}$ and $a \pm$ are defined in [37], $R_{0, n}$ are the reflection coefficients for the respective polarization for the 0 face or $\mathrm{n}$ face. $\Phi_{2}$ and $\Phi_{1}$ angles in (3) are depicted in Fig. 3.

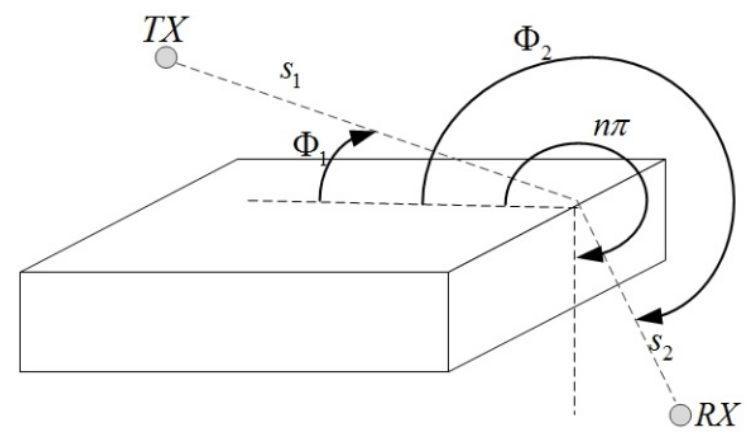

Fig. 3. Geometry for wedge diffraction coefficients.

The diffracted field is calculated by (4):

$$
E_{U T D}=e_{0} \frac{e^{-j k s_{1}}}{s_{1}} D^{\perp \|} \sqrt{\frac{s_{1}}{s_{2}\left(s_{1}+s_{2}\right)}} e^{-j k s_{2}}
$$

where $D^{\perp \|}$ are the diffraction coefficients in (3) and $s_{1}, s_{2}$ corresponds with the distances source-edge and edge-receiver point, respectively, as it is represented in Fig. 3. The path loss propagation is considered at each spatial point of the 3D scenario, considering the losses of propagation because of different material properties, at different distances $d$, with an attenuation constant $\alpha(\mathrm{Np} / \mathrm{m})$, and a phase constant $\beta(\mathrm{rad} / \mathrm{m})$. The total field is calculated considering all the incident vectorial fields inside each cuboid of the defined spatial mesh. Thus, the main principle of the RL techniques is that, for a given carrier frequency, at a specific bandwidth, where the materials are assumed to be spatially homogeneous and temporally non-dispersive in the band of interest, the impulse response of the channel can be determined. Consequently, taking into account this information, the wholly characterization of a stationary channel can be done.

The proposed simulation code has been extensively tested as a valid methodology to characterize electromagnetic propagation in complex scenarios [38-39], interference analysis [40] or electromagnetic dosimetry evaluation in wireless systems [41]. It has been demonstrated that the principle of GO/UTD gives precise radio wave propagation results when a whole $3 \mathrm{D}$ environment is taking into account. Nevertheless, the significant disadvantage of the algorithm is 
the high computational simulation time needed for complex and scenarios with great dimensions, which can be unaffordable in some occasions. In order to manage this issue, and achieve precise results with an affordable computational simulation time, the DE-GO technique has been analyzed and compared for different realistic scenarios, achieving good results. The DE technique neglect the edge contributions, and takes into account absorption and scattering electromagnetic phenomena caused by the obstacles. The main purpose of this work is to validate the power prediction tool in different complex and large scenarios, achieving precise results with an affordable computational time. The methodology followed is to compare the $\mathrm{GO} / \mathrm{DE}$ method with the complete technique GO/UTD/DE (which takes into account all the electromagnetic phenomena) in terms of accuracy and simulation time required, showing that the novel approach gives precise results with a relevant decrease of simulation time needed.

\section{DifFUSION EQUATION APPROACH}

\section{A. Ray Launching Modeling with Diffusion Equation}

It has been shown in the literature that in discrete random media with enough multiple scattering, electromagnetic waves have a diffusive behavior. Under the assumption of uniform scattering, the diffusion equation simplifies the equation of transfer in the classical transport theory [42].

Following the approach described in [11, 25, 43], it has been developed a new module to be implemented in the 3D $\mathrm{RL}$ tool. This new module is based on DE and considers absorption and scattering losses caused by obstacles. It can be implemented in both, the GO only approach and the GO/UTD technique. In this sense, the fully $\mathrm{GO} / \mathrm{UTD} / \mathrm{DE}$ methodology is the most precise approach because it considers all the electromagnetic phenomena encountered in a composite and heterogeneous scenario, such as reflection, refraction, diffraction and diffuse scattering. Nevertheless, the computational simulation time of this fully technique can be unaffordable for complex and large scenarios, as it is presented in the following sections. In this work, the comparison between different techniques for different scenarios is presented, showing that the GO/DE approach, which does not consider edge diffraction, achieve the best results. Although this technique does not take into account edge diffraction, it has been presented in the following sections that the impact of edge diffraction cannot be considered if the field is averaged taking into account all the obstacles (with the respective locations and orientations) within the whole $3 \mathrm{D}$ environment, conducting to an important decrease of computational time.

The GO/DE approach has been presented in [25]. The new technique is based on the statement that if the obstacles area density is higher than $10 \%$, then diffusion approaches can be applied to indoor scenarios [43]. The principle is that the whole 3D environment is divided in terms of horizontal and vertical 2D planes. Afterwards, the $2 \mathrm{D}$ planes with diffusive behavior are treated with the DE methodology, considering scattering and absorption losses due to obstacles.

The statistical average of the of the Poynting vector magnitude at any spatial location of the $3 \mathrm{D}$ scenario is the notion of specific intensity, in which the $\mathrm{DE}$ approach is based. In $2 \mathrm{D}$, the specific intensity can be described as a function of three input arguments: one angular coordinate $\xi$, providing the azimuthal direction of the average Poynting vector, and two spatial coordinates $\rho=(x, y) \equiv\left(r_{a}, \varnothing\right)$, where $r_{a}$ is the radial distance and $\emptyset$ is the azimuthal angle. The specific intensity, after normalization, is given by $I(\rho, \xi)=U_{d}(\rho)+\hat{\boldsymbol{s}} \overrightarrow{\boldsymbol{F}}(\rho) / \pi$, where $U_{d}$ is the average intensity (units of Watts $/ \mathrm{m}$ ), the flux density vector (units of Watts $/ \mathrm{m})$ is $\overrightarrow{\boldsymbol{F}}$ and $\hat{\boldsymbol{s}}=\widehat{\boldsymbol{x}} \cos \xi+\widehat{\boldsymbol{y}} \sin \xi$. Their relation is given by:

$$
\begin{aligned}
& U_{d}(\rho)=\frac{1}{2 \pi} \int_{0}^{2 \pi} I(\rho, s) d \xi \\
& \overrightarrow{\boldsymbol{F}}(\rho)=\frac{1}{2 \pi} \int_{0}^{2 \pi} \hat{\boldsymbol{s}} I(\rho, s) d \xi
\end{aligned}
$$

Following the formulation stated in [11], based on transport theory, the excess loss on a dB scale can be expressed as

$$
L_{e x}^{T}\left(r_{a}\right) \sim \frac{10 \sqrt{2} \sigma_{g} p_{0} \log e}{A_{0}} r_{a}-5 \log \left(\frac{\pi p_{0} \sigma_{g} r_{a}}{\sqrt{2} A_{0}}\right)
$$

where $p_{0}$ is the obstacle occupational density, $A_{0}$ is the average obstacle cross sectional area, $\sigma_{g}[\mathrm{~m}]$ is the geometrical cross section of the obstacles per unit length and $r_{a}$ is radial TX-RX distance.

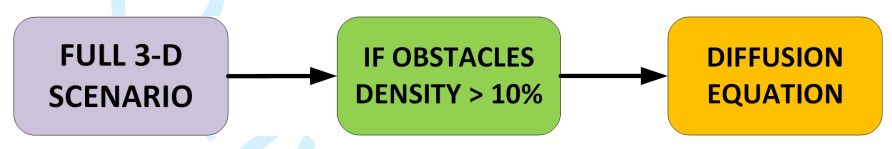

Fig. 4. Schematic view of the new considered approach.

For those horizontal or vertical planes which behave diffusively, the DE model has been applied, as it is shown in Fig. 4. As stated in section II, it is important to stress that the whole 3D scenario is divided into a grid, leading to a number of cuboids of fixed size. In the algorithm, we can define cuboid volume as input parameters. Depending of this cuboids size, the vertical and horizontal 2D planes are created, which could be different determined by the layout of the entire scenario. The methodology has been to calculate the obstacle density for each 2D vertical and horizontal plane. Afterwards, the planes with an obstacles area density bigger than $10 \%$ have been treated with DE. Considering the large size of obstacles when compared to the investigated wavelength, it can be assumed that the transmission and absorption cross sections are approximately equal to the geometric cross section [42]. 


\section{Simulation RESUltS AND VALIDATION OF THE ALGORITHM}

First, different simulations of typical complex indoor environments have been done with the different approaches. The considered scenarios are represented in Fig. 5, which shows three different environments: (a) an office environment, (b) a first floor of a house with eight rooms, and (c) a floor with several meeting rooms and offices. These three scenarios have been chosen because they represent typical complex indoor environments. All scenarios have several obstacles, such as tables of different dimensions and shapes, chairs, furniture, computers, shelves, etc. The dimensions of the scenarios are $(13 \mathrm{~m} \times 7 \mathrm{mx} 4.2 \mathrm{~m}), \quad(9 \mathrm{~m} \times 7.25 \mathrm{~m} \times 2.6 \mathrm{~m})$ and $(29.5 \mathrm{~m} \times 20.45 \mathrm{~m} \times 3.8 \mathrm{~m})$, respectively. Every obstacle within the scenarios have been considered in simulations, as well as the walls of different materials, which are also shown in the schematic view of the scenarios. The values of permittivity and conductivity employed in the simulated scenarios are defined in Table I [45].

TABLE I

RAY LAUNCHING MATERIAL PROPERTIES

\begin{tabular}{ccc}
\hline \hline Parameters & Permittivity $\left(\boldsymbol{\varepsilon}_{\mathbf{r}}\right)$ & Conductivity $(\boldsymbol{\sigma})[\mathbf{S} / \mathbf{m}]$ \\
\hline Air & 1 & 0 \\
Plywood & 2.88 & 0.21 \\
Brick wall & 4.11 & 0.0364 \\
Glass & 6.06 & $10^{-12}$ \\
Concrete & 5.66 & 0.142 \\
Metal & 4.5 & $4 * 10^{7}$ \\
Polycarbonate & 3 & 0.2 \\
\hline \hline
\end{tabular}

Table II presents the considered simulation input parameters for the GO/UTD/DE approach. The different cuboids resolution has been chosen according to a convergence analysis of cuboids size comparing with the dimensions of the scenarios [45]. The cuboid mesh resolution of the office environment generates to 84 horizontal XY-planes, 260 vertical YZ-planes and 140 vertical XZ-planes, the story house generates to 9 horizontal XY-planes, 25 vertical YZ-planes and 31 vertical XZ-planes and the third scenario generates to 4 horizontal XY-planes, 21 vertical YZ-planes and 30 vertical XZ-planes. In the case of the first scenario (office environment), only the horizontal plane that considers tables and chairs exceeds the obstacle area density of $10 \%$, with an obstacle density in this particular plane of $15.49 \%$. For the story house, several planes exceed the obstacle density of $10 \%$. In these $2 \mathrm{D}$ horizontal and vertical planes, DE have been applied in order to obtain precise results for those planes. The third scenario does not have any plane with an obstacle density larger than $10 \%$. Because of that, it has been treated also with DE to show that in this case the mean error is larger when DE is applied to those planes with a density smaller than $10 \%$. Thus, only those planes with a larger obstacle density than $10 \%$ must be treated with the DE approach [43].

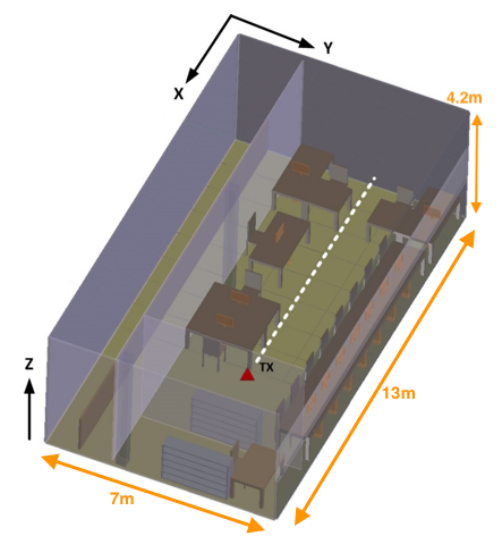

(a)

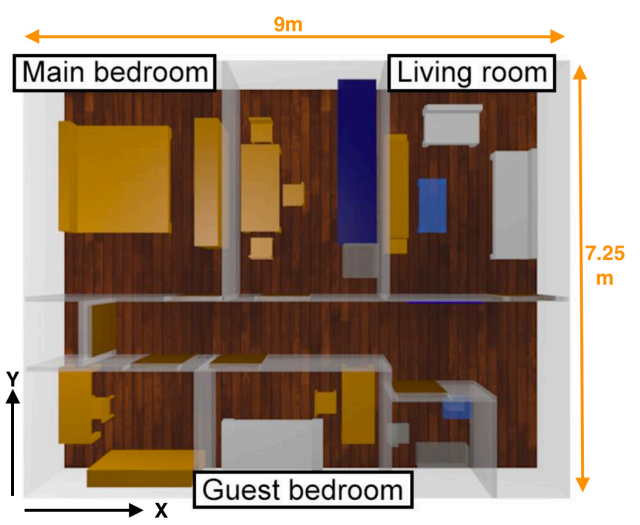

(b)

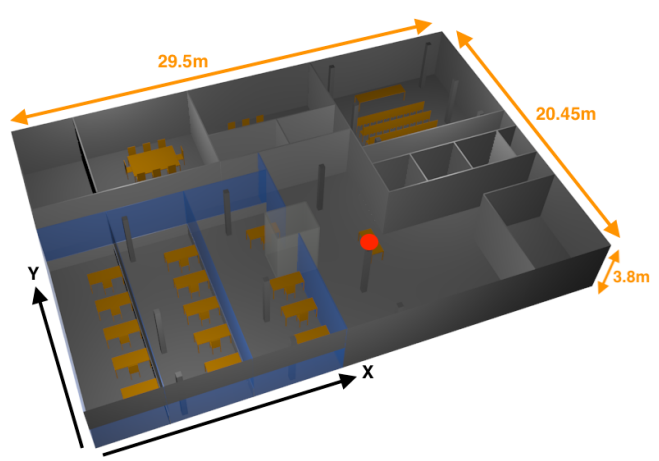

(c)

Fig. 5. Aerial view of the considered scenarios: (a) typical office environment (b) first floor of a house (c) floor with several meeting rooms and offices. Ceiling has not been shown for illustration.

TABLE II

SIMULATION PARAMETERS IN THE RAY LAUNCHING SOFTWARE

$\begin{array}{cc}\text { Frequency } & 2.4 \mathrm{GHz} \\ \text { Transmitter power } & 0 \mathrm{dBm} \\ \text { Antenna gain } & 5 \mathrm{dBi} \\ \text { al plane angle resolution }(\Delta \Phi) & 1^{\circ} \\ \text { plane angle resolution }(\Delta \theta) & 1^{\circ} \\ \text { Reflections } & 7\end{array}$

Cuboids resolution

$5 \mathrm{~cm} / 30 \mathrm{~cm} / 1 \mathrm{~m}$ 


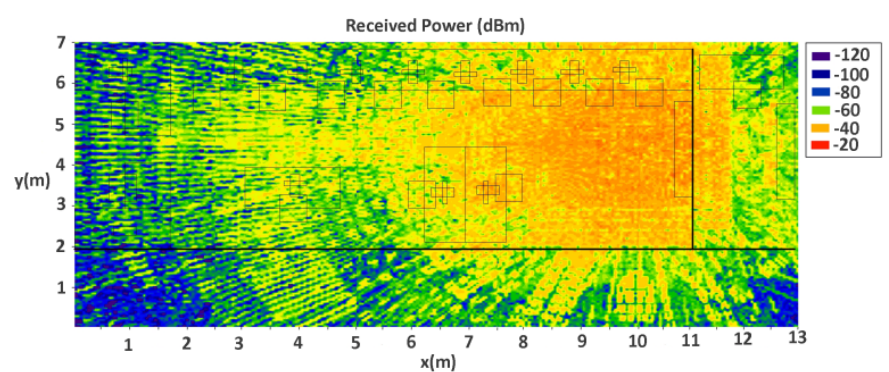

Fig. 6. XY plane of received power $(\mathrm{dBm})$ for $0.8 \mathrm{~m}$ height taking into account all the furniture in the office environment.

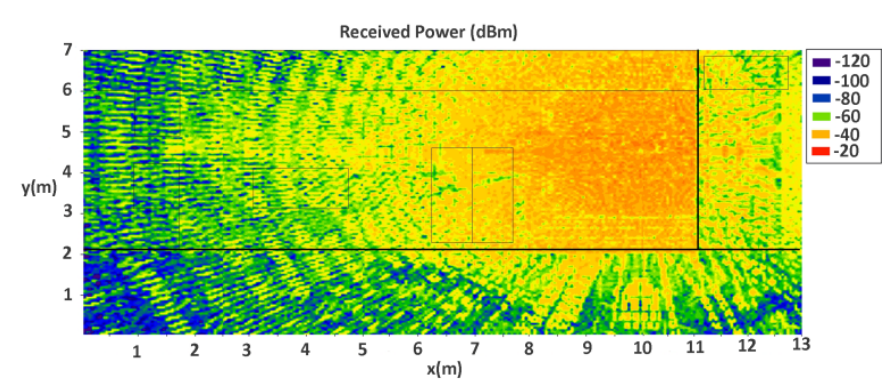

Fig. 7. XY plane of received power $(\mathrm{dBm})$ for $0.8 \mathrm{~m}$ height taking into account only the tables in the office environment.

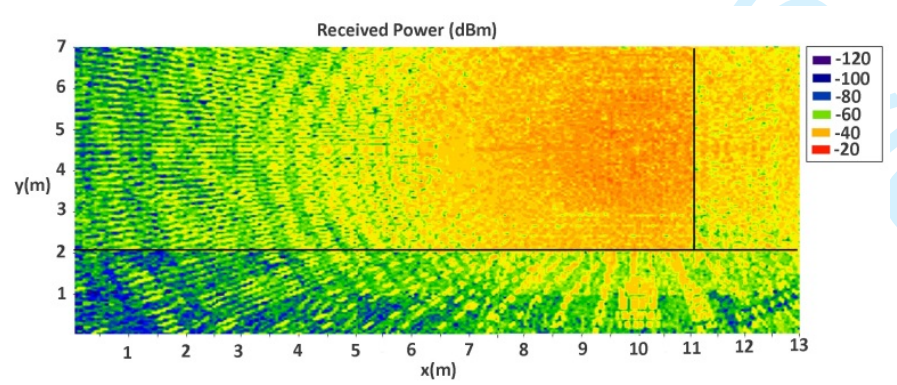

Fig. 8. XY plane of received power $(\mathrm{dBm})$ for $0.8 \mathrm{~m}$ height without obstacles in the office environment.

Firstly, simulations have been done in the office environment scenario, where the impact of furniture has been assessed. The impact of the furniture in the radio wave propagation in indoor scenarios has a large impact in the weakening of the signal. Fig. 6, 7 and 8 show the XY-planes of received power for a height of $0.8 \mathrm{~m}$. For these simulations, the GO/UTD/DE fully approach has been used, and results show that the high influence of furniture in the environment related with radio wave propagation, showing that when more obstacles are taking into account, the interference is bigger.

Fig. 9 shows the received power distribution as a function of obstacle density variation, along the $\mathrm{X}$-axis at $\mathrm{Y}=5.75 \mathrm{~m}$. Higher fading losses are observed as the propagating paths encounter higher furniture density, as expected.

The objective is to assess the impact of the scattering in this complex indoor scenario. For that purpose, several simulations have been developed with the implementation of the DE modeling. Then, a comparison has been made between the GO only approach, the GO with edge contributions and full method considering GO/UTD/DE. The comparison of received power for the different techniques along the $\mathrm{X}$-axis for $\mathrm{Y}=4.5 \mathrm{~m}$ is shown in Fig. 10 and Fig. 11. Fig. 10 presents the comparison between GO/UTD and GO/DE approaches. It can be seen that the edge contributions can enhance the channel performance in certain points as the received power is higher on the whole than the GO/DE approach. However, the scattering and absorption due to obstacles, which is always present in practical situations, reduces the received power in almost every spatial point of the space. In the light of this, it can be concluded that is really important to take into account scattering phenomena in order to achieve precise results to characterize adequately the wireless channel.

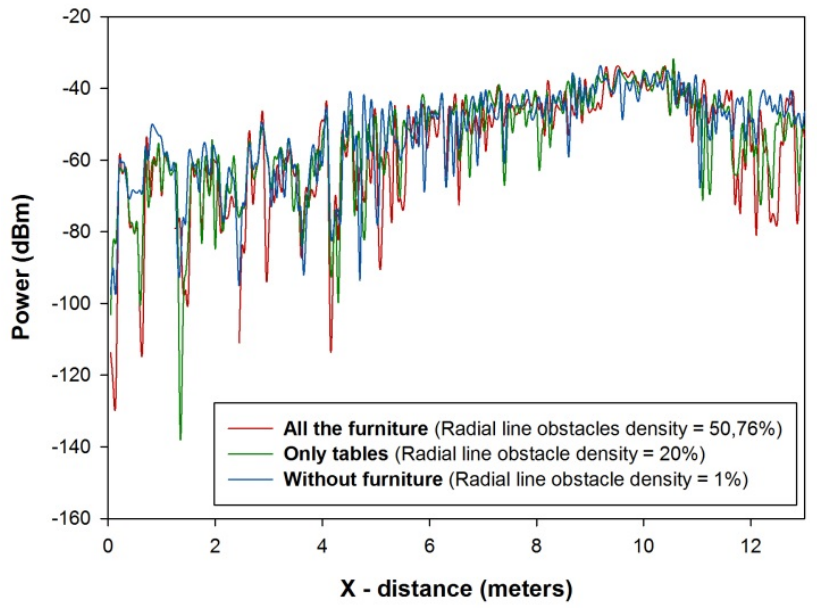

Fig. 9. Distribution of received power for the three cases considered: all the furniture, only tables and without furniture in the office environment.

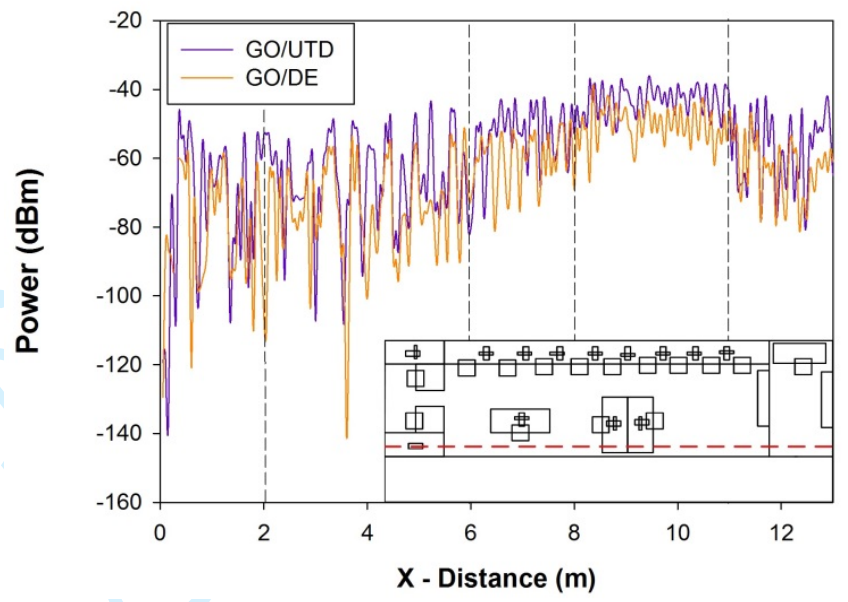

Fig. 10. Comparison between received power lines in $\mathrm{dBm}$ for the GO/UTD and $\mathrm{GO} / \mathrm{DE}$ methods in the office environment.

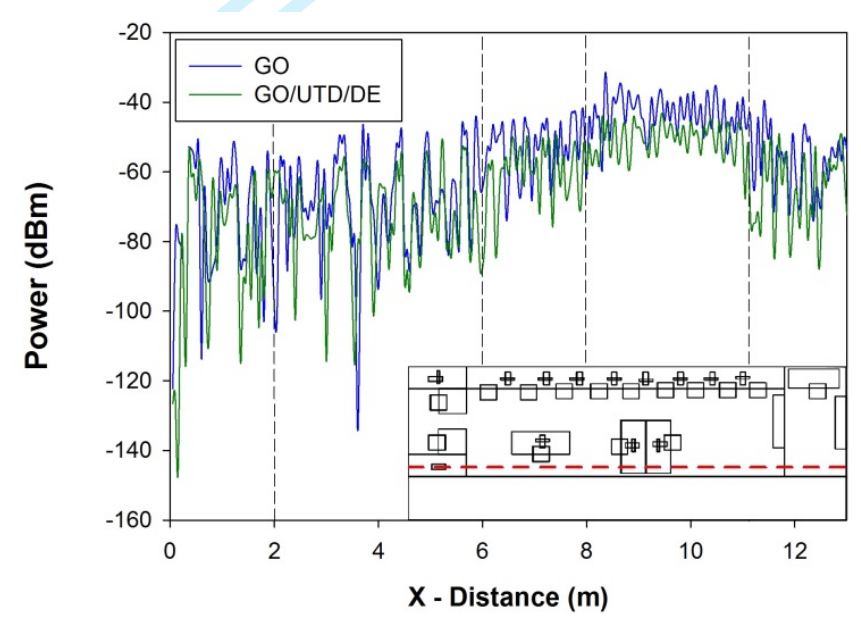

Fig. 11. Comparison between received power lines in $\mathrm{dBm}$ for the GO only approach and the fully GO/UTD/DE method in the office environment.

Fig. 11 presents the comparison between GO only approach and the full $\mathrm{GO} / \mathrm{UTD} / \mathrm{DE}$ technique. It can be observed that the GO only approach predicts bigger levels of received power along the entire radial. However, the full method which 
considers into account all electromagnetic phenomena predicts lower values because it considers more losses mainly due to scattering and diffraction, showing higher accuracy. The figure also depicts the location of obstacles, given by the vertical dashed lines, in which larger variability in estimated losses can be observed.

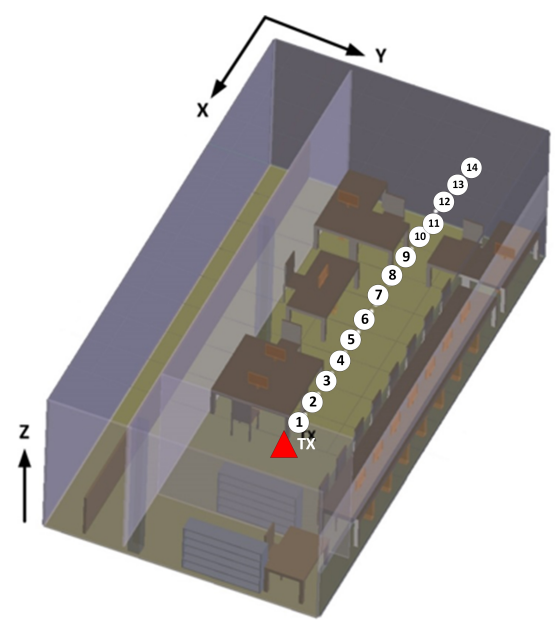

(a)

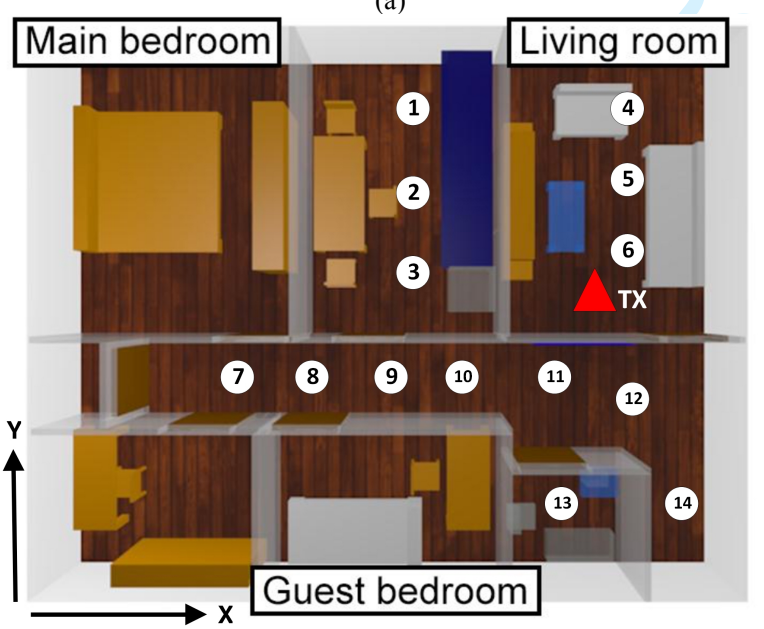

(b)

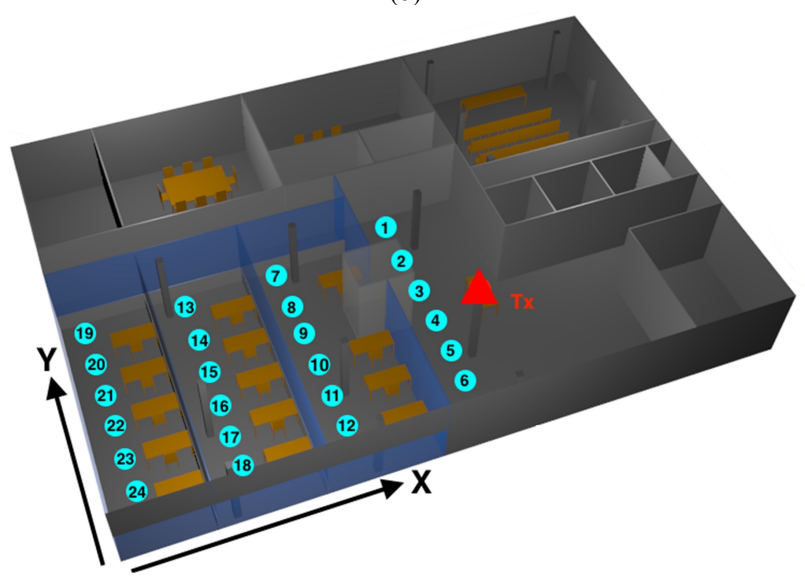

(c)

Fig. 12. Measurement points for the scenarios considered: (a) office environment (b) one-story house and (c) floor with different rooms with the position of the transmitter.

\section{Measurement Results And Discussion}

A campaign of real measurements in the three scenarios considered has been done in order to validate the results previously shown. For that purpose, the methodology has been to connect a transmitter antenna to a signal generator at $2.4 \mathrm{GHz}$ frequency. The transmitter antenna has been placed at the coordinates depicted with a red triangle in Fig. 12 in the three scenarios considered. A portable Agilent N1996A has been employed as signal generator and an Agilent N9912 Field Fox as spectrum analyzer. Both antennas, transmitter and receiver, are omnidirectional antennas (Picea $2.4 \mathrm{GHz}$ Swivel Atennas from Antenanova). Measurements have been performed in the measurement points represented in Fig. 12 for the three scenarios respectively, at a height of $0.80 \mathrm{~m}$ in the three cases.

Simulation and measurement results for the different simulation techniques are represented in Fig. 13 for the first two scenarios, which have planes with obstacles densities higher than $10 \%$. For the three scenarios considered, the bandwidth considered for the measurements were $100 \mathrm{MHz}$, and the measurement time was 60 seconds at each spatial point, considering the peak power detected values. The comparison has been made for the same spatial samples with the received power estimated by simulation and by the real measurements. From Fig. 13, it can be seen that all the proposed methods follow the received power measurement trend.

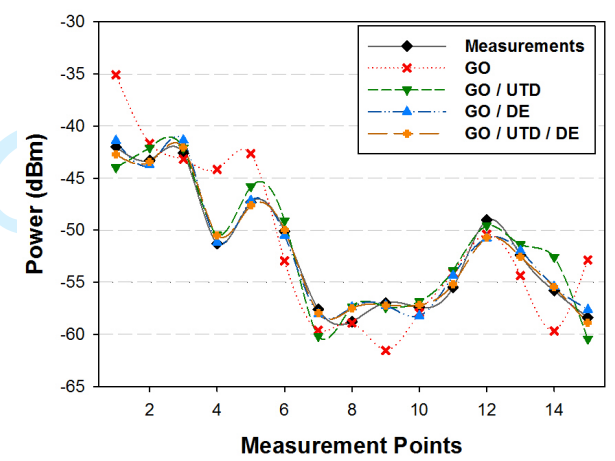

(a)

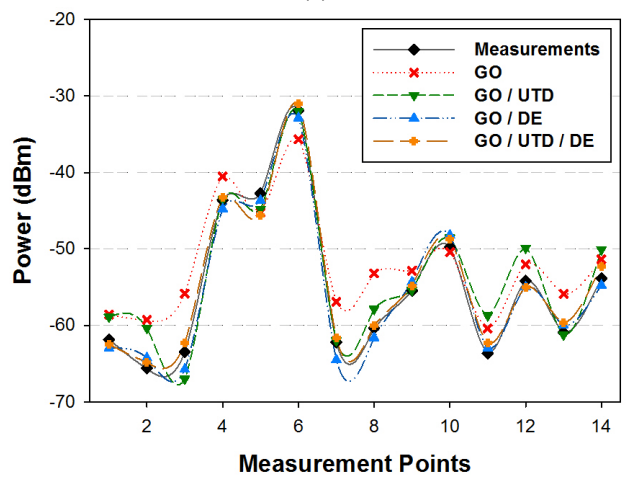

(b)

Fig. 13. Comparison between the 3D RL simulation results and real measurements for $2.4 \mathrm{GHz}$ frequency for the different simulation approaches: (a) Office environment (b) One-story house. 
TABLE III

MEAN ERROR AND STD. DEVIATION FOR THE DIFFERENT APPROACHES

\begin{tabular}{|c|c|c|c|c|c|c|}
\hline & & & $\begin{array}{c}\text { CASE } \\
1\end{array}$ & CASE 2 & CASE 3 & CASE 4 \\
\hline & Obstacles Density & & GO & GO+UTD & GO+DE & GO+UTD+DE \\
\hline \multirow{2}{*}{$\begin{array}{c}\text { Office } \\
\text { environment }\end{array}$} & \multirow{2}{*}{$>10 \%$} & Mean error $(\mathrm{dB})$ & 3.01 & 1.37 & 0.89 & 0.62 \\
\hline & & Std. Deviation (dB) & 2.30 & 0.81 & 0.96 & 0.73 \\
\hline \multirow{2}{*}{$\begin{array}{c}\text { One-Story } \\
\text { house }\end{array}$} & \multirow{2}{*}{$>10 \%$} & Mean error (dB) & 2.30 & 1.30 & 1.23 & 1.01 \\
\hline & & Std. Deviation (dB) & 2.04 & 1.91 & 0.49 & 0.65 \\
\hline \multirow{2}{*}{$\begin{array}{c}\text { Floor with } \\
\text { several rooms }\end{array}$} & \multirow[b]{2}{*}{$<10 \%$} & Mean error $(\mathrm{dB})$ & 2.86 & 1.89 & 8.29 & 9.09 \\
\hline & & Std. Deviation (dB) & 1.98 & 1.44 & 9.27 & 11.19 \\
\hline
\end{tabular}

Table III shows the mean error and standard deviation between the different approaches for the three considered scenarios, the first two with planes with an obstacle density larger than $10 \%$ and the third one, with obstacles density smaller than $10 \%$. It can be seen that the full GO/UTD/DE technique is the most accurate for the first two cases with a mean error of $0.62 \mathrm{~dB}$ and a standard deviation of $0.73 \mathrm{~dB}$ for the office environment and a mean error of $1.01 \mathrm{~dB}$ and a standard deviation of $0.65 \mathrm{~dB}$ for the story house. However, it is observed that in these two scenarios, that for the GO/DE approach, the mean error only increases by $0.27 \mathrm{~dB}$ in the case of the office environment, and $0.22 \mathrm{~dB}$ in the case of the onestory house, while the computational time is reduced $40 \%$ and $90 \%$ respectively, with respect to the full $\mathrm{GO} / \mathrm{UTD} / \mathrm{DE}$ approach. Fig. 14 shows the computational time required for the four considered techniques, for the first two scenarios considered. It can be seen from Fig. 14 the inclusion of the analysis of the diffracted rays takes a large amount of time. However, the novel technique GO/DE, which does not take into account the impact of edges because it considers the averaged received power field due to the different obstacles in the whole $3 \mathrm{D}$ environment, produces reasonable results. Table III shows the mean error and standard deviation for the third scenario considered, which does not have any plane with an obstacle density larger than $10 \%$. It can be seen that the mean error and standard deviation for the fully $\mathrm{GO} / \mathrm{UTD} / \mathrm{DE}$ approach and the novel technique $\mathrm{GO} / \mathrm{DE}$ increase significantly in this third scenario. Thus, it is shown that DE approach does not work in this scenario, with planes with obstacles densities smaller than $10 \%$, as it is shown in [43]. It can be concluded that it is highly important to achieve a tradeoff between precision of the results and simulation computational time, when these hybrid approaches are employed. In this sense, it is shown that the novel technique $\mathrm{GO} / \mathrm{DE}$ is precise for a complex indoor environment while a considerable amount of computational time is reduced, when compared with the fully $\mathrm{GO} / \mathrm{UTD} / \mathrm{DE}$ approach.

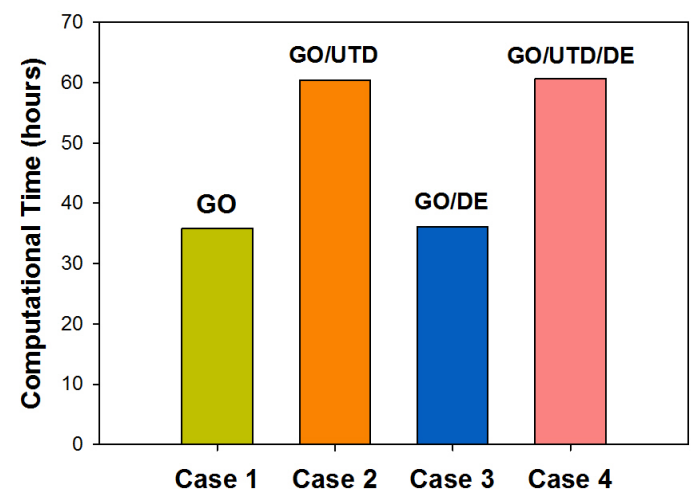

(a)

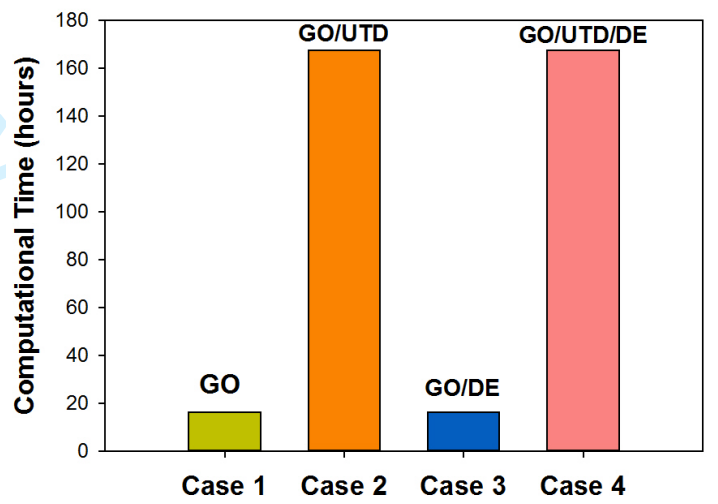

(b)

Fig. 14. Computational time required for the complete simulation of the considered scenarios for the different simulation approaches: (a) Office environment (b) One-story house.

\section{CONCLUSION}

In this work, a new technique which combines a 3-D Ray Launching with a Diffusion Equation (GO/DE) method based on the transport equation has been presented and validated in different realistic complex indoor environments. The new approach has been compared with the reference solution, Geometrical Optics with edge contributions and Diffusion equation $(\mathrm{GO} / \mathrm{UTD} / \mathrm{DE})$, in different simulations of real scenarios. The results show that there is a small increase in mean error values when applying the combined approaches, in the order of $1 \mathrm{~dB}$. The new approach considerably decreases 
computational saving, up to $90 \%$ in simulation time, with similar accuracy levels.

Currently, a full 3D approach of scattering and absorption losses handled by diffusion equation is being considered, with the aim of not needing to take into account the orthogonal 2D planes. This will be presented in future work.

\section{ACKNOWLEDGMENTS}

The authors also acknowledge the support received from the Ministry of Economy and Competitiveness, Government of Spain, under grant TEC2013-45585-C2-1-R.

\section{REFERENCES}

[1] Rappaport, T. Wireless communications: principles and practice.; Ed.; Communications Engineering and Emerging Technologies Series.; 2nd ed.; Prentice Hall PTR, Upper Saddle River, NJ: United States, 2002.

[2] J. Kivinen, Xiongwen Zhao and P. Vainikainen, "Empirical characterization of wideband indoor radio channel at $5.3 \mathrm{GHz}$, in IEEE Trans on Ant and Prop, vol. 49, no. 8, pp. 1192-1203, Aug 2001.

[3] G. Durgin, T. S. Rappaport and Hao Xu, "Measurements and models for radio path loss and penetration loss in and around homes and trees at $5.85 \mathrm{GHz}, "$ in IEEE Transactions on Communications, vol. 46, no. 11, pp. 1484-1496, Nov 1998.

[4] M. I. S. Chowdhury, W. Zhang and M. Kavehrad, "Combined Deterministic and Modified Monte Carlo Method for Calculating Impulse Responses of Indoor Optical Wireless Channels," in Journal of Lightwave Technology, vol. 32, no. 18, pp. 3132-3148, Sept.15, 152014.

[5] V. Degli-Esposti et al., "A Semi-Deterministic Model for Outdoor-toIndoor Prediction in Urban Areas," in IEEE Antennas and Wireless Propagation Letters, vol. 16, pp. 2412-2415, 2017.

[6] Y. Wu, Z. Chen, W. Fan, J. Wang and J. Li, "A Wave-Equation-Based Spatial Finite-Difference Method for Electromagnetic Time-Domain Modeling," in IEEE Antennas and Wireless Propagation Letters, vol. 17, no. 5, pp. 794-798, May 2018.

[7] I. P. Shkarofsky and S. B. Nickerson, "Computer modeling of multipath propagation: Review of ray-tracing techniques," in Radio Science, vol. 17, no. 05, pp. 1133-1158, Sept.-Oct. 1982.

[8] A. G. Kanatas and P. Constantinou, "A propagation prediction tool for urban mobile radio systems," in IEEE Transactions on Vehicular Technology, vol. 49, no. 4, pp. 1348-1355, Jul 2000.

[9] K. W. Kim and S. J. Oh, "Geometric Optics-Based Propagation Prediction Model in Urban Street Canyon Environments," in IEEE Antennas and Wireless Propagation Letters, vol. 15, pp. 1128-1131, 2016.

[10] D. Ullmo and H. U. Baranger, "Wireless propagation in buildings: A statistical scattering approach," IEEE Trans. Veh. Technol., vol. 48, pp. 947-955, 1999.

[11] R. Janaswamy, "An indoor pathloss model at $60 \mathrm{GHz}$ based on transport theory," IEEE Antennas Wireless Propag. Letters, vol. 5, pp. 58-60, 2006.

[12] Y. Corre and Y. Lostanlen, "Three-Dimensional Urban EM Wave Propagation Model for Radio Network Planning and Optimization Over Large Areas," in IEEE Transactions on Vehicular Technology, vol. 58, no. 7, pp. 3112-3123, Sept. 2009.

[13] E. M. Vitucci, F. Mani, V. Degli-Esposti and C. Oestges, "Polarimetric Properties of Diffuse Scattering From Building Walls: Experimental Parameterization of a Ray-Tracing Model," in IEEE Trans. on Ant. and Prop., vol. 60, no. 6, pp. 2961-2969, June 2012.

[14] F. Fuschini, H. El-Sallabi, V. Degli-Esposti, L. Vuokko, D. Guiducci and P. Vainikainen, "Analysis of Multipath Propagation in Urban Environment Through Multidimensional Measurements and Advanced Ray Tracing Simulation," in IEEE Trans. on Ant. and Prop., vol. 56, no. 3, pp. 848-857, March 2008.

[15] J. H. Tarng, Ruey-Shan Chang, Jiunn-Ming Huang and Yih-Min Tu, "A new and efficient hybrid model for estimating space diversity in indoor environment," in IEEE Transactions on Vehicular Technology, vol. 49, no. 2, pp. 457-466, Mar 2000.
[16] L. Azpilicueta, M. Rawat, K. Rawat, F. Ghannouchi, F. Falcone, “A Ray Launching-Neural Network Approach for Radio Wave Propagation Analysis in Complex Indoor Environments," IEEE Trans. on Ant. and Prop., vol. 62, nº 5, May 2014.

[17] F. Saez de Adana, O. Gutierrez Blanco, I. Gonzalez Diego, J. Perez Arriaga and M. F. Catedra, "Propagation model based on ray tracing for the design of personal communication systems in indoor environments," in IEEE Transactions on Vehicular Technology, vol. 49, no. 6, pp. 21052112, Nov 2000.

[18] J. P. Rossi, J. P. Barbot and A. J. Levy, "Theory and measurement of the angle of arrival and time delay of UHF radiowaves using a ring array," in IEEE Trans on Ant and Prop, vol. 45, no. 5, pp. 876-884, May 1997.

[19] Y. Corre and Y. Lostanlen, "Three-Dimensional Urban EM Wave Propagation Model for Radio Network Planning and Optimization Over Large Areas," in IEEE Transactions on Vehicular Technology, vol. 58, no. 7, pp. 3112-3123, Sept. 2009.

[20] H. M. El-Sallabi, G. Liang, H. L. Bertoni, I. T. Rekanos and P. Vainikainen, "Influence of diffraction coefficient and corner shape on ray prediction of power and delay spread in urban microcells," in IEEE Trans on Ant and Prop, vol. 50, no. 5, pp. 703-712, May 2002.

[21] C. H. Durney, D. A. Christensen and R. W. Grow, "A mathematical technique for an exact small-signal field analysis of multiple-stream interaction in a finite longitudinal magnetic field," in IEEE Transactions on Electron Devices, vol. 16, no. 7, pp. 609-615, Jul 1969.

[22] Z. Zhijun and Y. Wei, "Damage of Interconnects by Electromigration Induced Surface Evolution," in Tsinghua Science and Technology, vol. 2, no. 2, pp. 574-577, June 1997.

[23] G. Wolfle, R. Wahl, P. Wertz, P. Wildbolz and F. Landstorfer, "Deterministic Propagation Model for the Planning of Hybrid Urban and Indoor Scenarios," 2005 IEEE 16th International Symposium on Personal, Indoor and Mobile Radio Communications, Berlin, 2005, pp. 659-663.

[24] F. Fuschini, E. M. Vitucci, M. Barbiroli, G. Falciasecca and V. DegliEsposti, "Ray tracing propagation modeling for future small-cell and indoor applications: A review of current techniques," in Radio Science, vol. 50, no. 6, pp. 469-485, June 2015.

[25] L. Azpilicueta, F. Falcone and R. Janaswamy, "A Hybrid Ray Launching-Diffusion Equation Approach for Propagation Prediction in Complex Environments," IEEE Antennas and Wireless Propagation Letters, vol. 16, 2017.

[26] A. Cardama, "Antenas", Editions UPC, 1993.

[27] H. D. Hristov, R. Feick, W. Grote and P. Fernandez, "Indoor signal focusing by means of Fresnel zone plate lens attached to building wall," in IEEE Transactions on Antennas and Propagation, vol. 52, no. 4, pp. 933-940, April 2004.

[28] J. Keller, "Diffraction of a convex cylinder," in IRE Trans. on Antennas and Prop., vol. 4, no. 3, pp. 312-321, July 1956.

[29] I. Kupiec, L. B. Felsen, S. Rosenbaum, J. B. Keller and P. Chow, "Reflection and transmission by a random medium," in Radio Science, vol. 4, no. 11, pp. 1067-1077, Nov. 1969.

[30] M. C. Liang, C. W. Chuang and P. H. Pathak, "A generalized uniform geometrical theory of diffraction ray solution for the diffraction by a wedge with convex faces," in Radio Science, vol. 31, no. 4, pp. 679-691, July-Aug. 1996.

[31] H. G. Booker and P. C. Clemmow, "A relation between the Sommerfeld theory of radio propagation over a flat earth and the theory of diffraction at a straight edge," in Electrical Engineers, Journal of the Institution of, vol. 1950, no. 3, pp. 78-79, March 1950.

[32] G. Deschamps, J. Boersma and Shung-Wu Lee, "Three-dimensional half-plane diffraction: Exact solution and testing of uniform theories," in IEEE Trans. on Antennas and Prop., vol. 32, no. 3, pp. 264-271, Mar 1984.

[33] K. Allsebrook and J. D. Parsons, "Mobile radio propagation in British cities at frequencies in the VHF and UHF bands," in IEEE Transactions on Vehicular Technology, vol. 26, no. 4, pp. 313-323, Nov. 1977.

[34] F. Ikegami, T. Takeuchi and S. Yoshida, "Theoretical prediction of mean field strength for urban mobile radio," in IEEE Trans on Ant and Prop, vol. 39, no. 3, pp. 299-302, Mar 1991.

[35] C. Tornevik, J. E. Berg, F. Lotse and M. Madfors, "Propagation models, cell planning and channel allocation for indoor applications of cellular systems," IEEE 43rd Vehicular Technology Conference, Secaucus, NJ, 1993, pp. 867-870. 
[36] S. Y. Seidel, T. S. Rappaport and R. Singh, "Path loss and multipath delay statistics in four European cities for $900 \mathrm{MHz}$ cellular and microcellular communications," in Electronics Letters, vol. 26, no. 20, pp. 1713-1715, 27 Sept. 1990.

[37] R. J. Luebbers, "Comparison of lossy wedge diffraction coefficients with application to mixed path propagation loss prediction," in IEEE Transactions on Antennas and Propagation, vol. 36, no. 7, pp. 10311034, Jul 1988.

[38] L. Azpilicueta, J. J. Astrain, P. Lopez-Iturri, F. Granda, C. VargasRosales, J. Villadangos, A. Perallos, A. Bahillo, F. Falcone, "Optimization and Design of Wireless Systems for the Implementation of Context Aware Scenarios in Railway Passenger Vehicles," IEEE Transactions on Intelligent Transportation Systems, vol. 18, no. 10, October 2017, pp. 2838-2850.

[39] L. Azpilicueta, P. López-Iturri, E.Aguirre, C. Martínez, J. J. Astráin, J. Villadangos and F. Falcone, "Evaluation of Deployment Challenges of Wireless Sensor Networks at Signalized Intersections," Sensors 2016, 16(7), 1140.

[40] A. Córdoba et al., "SesToCross: Semantic Expert System to Manage Single-Lane Road Crossing," in IEEE Transactions on Intelligent Transportation Systems, vol. 18, no. 5, pp. 1221-1233, May 2017.

[41] F. Casino, L. Azpilicueta, P. Lopez-Iturri, E. Aguirre, F. Falcone and A. Solanas, "Optimized Wireless Channel Characterization in Large Complex Environments by Hybrid Ray Launching-Collaborative Filtering Approach," in IEEE Antennas and Wireless Propagation Letters, vol. 16, pp. 780-783, 2017.

[42] A. Ishimaru, Wave Propagation and Scattering in Random Media. New York: Academic, 1978, vol. 1.

[43] J. Xu and R. Janaswamy, "On the Diffusion of Electromagnetic Waves and Applicability of Diffusion Equation to Multipath Random Media," IEEE Transactions of Antennas and Propagation, vol. 56, $\mathrm{n}^{\circ} 4$, April 2008.

[44] A. Constantine Balanis. Advanced engineering electromagnetics. Vol. 205. Wiley New York, 1989.

[45] L. Azpilicueta, P. Lopez-Iturri, E. Aguirre, C. Vargas-Rosales, A. León, F. Falcone, "Influence of Meshing Adaption in Convergence Performance of Deterministic Ray Launching Estimation in Indoor Scenarios," Journal of Electromagnetic Waves and Applications, 2017, $31: 5,544-559$.

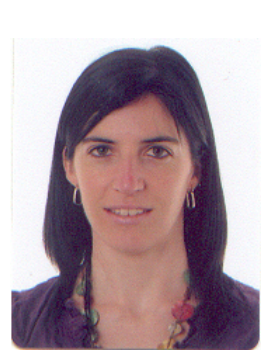

Leyre Azpilicueta (M15) received her Telecommunications Engineering Degree (2009), her Master Degree of Communications (2011) and her $\mathrm{Ph} . \mathrm{D}$. Degree in Telecommunication Technologies (2015), at the Public University of Navarre (UPNa), in Spain. In 2010 she worked in the R\&D department of RFID Osés as radio engineer. She is currently working as Associate Professor and Researcher at Tecnologico de Monterrey, Campus Monterrey, Mexico. Her research interests are on radio propagation, mobile radio systems, ray tracing and channel modeling. She has over 110 contributions in relevant journals and conference publications. She has been recipient of the Best PhD in 2016 awarded by the Colegio Oficial de Ingenieros de Telecomunicación, the 'IEEE Antennas and Propagation Society Doctoral Research Award 2014', and the
'Young Professors and Researchers Santander Universities 2014 mobility award'

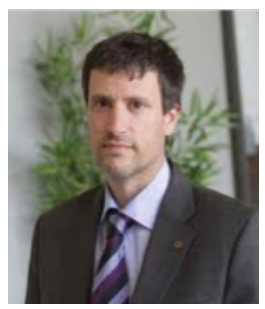

Francisco Falcone (M05, SM09) received his Telecommunication Engineering Degree (1999) and $\mathrm{PhD}$ in Communication Engineering (2005), both at the Public University of Navarre in Spain. From 1999 to 2000 he worked as Microwave Commissioning Engineer, Siemens-Italtel. From 2000 to 2008 he worked as Radio Network Engineer, Telefónica Móviles. In 2009 he co-founded Tafco Metawireless. From 2003 to 2009 he was also Assistant Lecturer at UPNA, becoming Associate Professor in 2009. His research area is artificial EM media, complex EM scenarios and wireless system analysis.

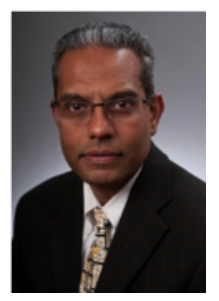

Ramakrishna Janaswamy received his Ph.D. degree in electrical engineering in 1986 from the University of Massachusetts, Amherst. He received his Master's degree in Microwave and Radar Engineering from IITKharagpur, India in 1983 and the Bachelor's degree in Electronics and Communications Engineering from REC-Warangal (now NIT-Warangal), India in 1981. From August 1986 to May 1987, he was an Assistant Professor of electrical engineering at Wilkes University, Wilkes Barre, PA. From August 1987August 2001 he was on the faculty of the Department of Electrical and Computer Engineering, Naval Postgraduate School, Monterey, CA. In September 2001 he joined the Department of Electrical \& Computer Engineering, University of Massachusetts, Amherst, where he is a currently a Professor. $\mathrm{He}$ was a visiting researcher at the Center for PersonKommunikation, Aalborg, Denmark from September 1997 to June 1998 and spent the Summers of 1994 and 1995 at SPAWARSYSCEN, San Diego, California and NASA Ames Research Center, Moffett Field, California, respectively. His research interests include deterministic and stochastic radio wave propagation modeling, analytical and computational electromagnetics, antenna theory and design, and wireless communications. Rama Janaswamy is a Fellow of IEEE and was the recipient of the R. W. P. King Prize Paper Award of the IEEE Transactions on Antennas and Propagation in 1995. For his services to the IEEE Monterey Bay Subsection, he received the IEEE 3rd Millennium Medal from the Santa Clara Valley Section in 2000. He is an elected member of U.S. National Committee of International Union of Radio Science, Commissions B and F. He served as an Associate Editor of Radio Science from January 1999-January 2004 and Associate Editor of IEEE Transactions on Vehicular Technology from 20032006. He is currently an Associate Editor of IEEE Transactions on Antennas and Propagation and of the IETE (India) Technical Reviews. He is the author of the book Radiowave Propagation and Smart Antennas for Wireless Communications, Kluwer Academic Publishers, November 2000 and a contributing author in Handbook of Antennas in Wireless Communications, L. Godara (Ed.), CRC Press, August 2001 and Encyclopedia of RF and Microwave Engineering, K. Chang (Ed.), John Wiley \& Sons, 2005. 